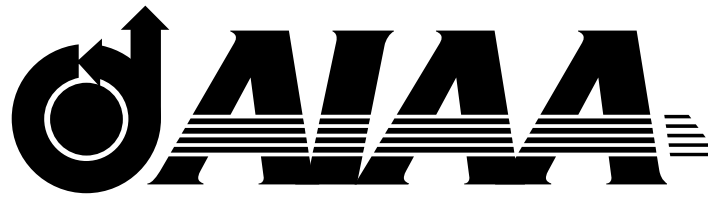

\section{AIAA 2004-1181}

\section{The Effect of Compressibility on}

Rarefied Hypersonic Flow over Power Law Leading Edges

Wilson F. N. Santos

National Institute for Space Research, Cachoeira Paulista, SP 12630-000 Brazil

Mark J. Lewis

University of Maryland, College Park, MD 20742

42nd AIAA Aerospace Sciences Meeting and Exhibit

January 5-8, 2004/Reno, NV

For permission to copy or republish, contact the American Institute of Aeronautics and Astronautics 1801 Alexander Bell Drive, Suite 500, Reston, VA 20191-4344 


\title{
The Effect of Compressibility on Rarefied Hypersonic Flow over Power Law Leading Edges
}

\author{
Wilson F. N. Santos* \\ National Institute for Space Research, Cachoeira Paulista, SP 12630-000 Brazil \\ Mark J. Lewis ${ }^{\dagger}$ \\ University of Maryland, College Park, MD 20742
}

\begin{abstract}
Hypersonic flow past power law shaped leading edges at zero incidence is investigated for a range of freestream Mach number from 5 to 12. The simulations were performed by using a Direct Simulation Monte Carlo Method. A method that has demonstrated to yield excellent comparison with experimental data, and that properly accounts for thermal nonequilibrium effects that arise near the leading edge and that are especially important at high Mach number. The work is motivated by interest in assessing the overall performance of power-law shaped leading edges in order to consider them as possible candidates for blunting geometries of hypersonic leading edges. The results presented highlight the sensitivity of the heat transfer coefficient, the total drag coefficient, and the shock wave standoff distance to changes on the freestream Mach number.
\end{abstract}

\section{Nomenclature}

Drag coefficient, Eq. (6)

Pressure drag coefficient

thermal velocity, $\mathrm{m} / \mathrm{s}$

Drag force, $\mathrm{N}$

specific energy, $\mathrm{J} / \mathrm{kg}$

Knudsen number

Body length, m

Mach number

Molecular mass, kg

Number of molecules

Body power law exponent

Pressure, $\mathrm{N} / \mathrm{m}^{2}$

Heat flux, $\mathrm{W} / \mathrm{m}^{2}$

Reynolds number

Arc length, m

Temperature, K

Velocity, m/s

Specific heat ratio

\section{Constant in power-law body equation}

Skin friction drag coefficient

$$
\text { Heat transfer coefficient, Eq. }
$$

Body height at the base, $\mathrm{m}$

Dimensionless arc length $\left(=s / \lambda_{\infty}\right)$

Cartesian axes in physical space, $\mathrm{m}$

Shock wave standoff distance, $\mathrm{m}$

Coordinate normal to body surface, $\mathrm{m}$

Wedge half angle, body slope angle, deg

${ }^{*}$ Researcher, Combustion and Propulsion Laboratory. AIAA Member.

${ }^{\dagger}$ Professor, Aerospace Engineering Department. AIAA Associate Fellow.

Copyright (c) 2004 by the American Institute of Aeronautics and Astronautics, Inc. All rights reserved.

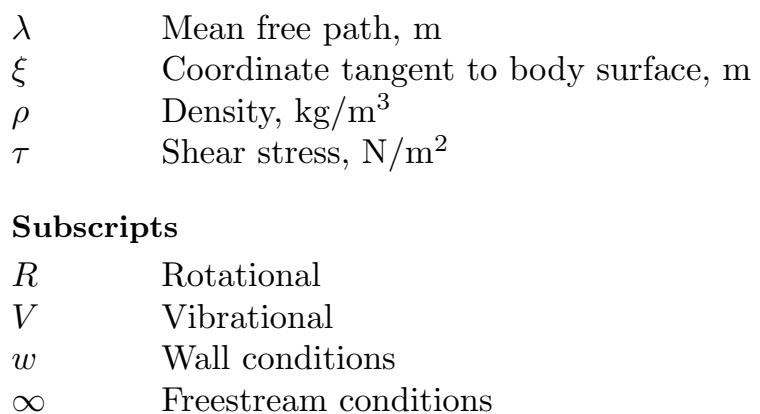

\section{Introduction}

A $\mathrm{N}$ efficient design of future airbreathing hypersonic vehicles will depend on high-lift, low-drag configurations in order to overcome the aerodynamic forces involved in high-speed flight. For this purpose, waveriders, first conceived by Nonweiler ${ }^{1}$, have been considered as one of the promising vehicle concepts under consideration. Waveriders are vehicles that are designed so that the bow shock is everywhere attached to the sharp leading edge. Because the shock wave is attached to the leading edge of the vehicle, the upper and lower surfaces of the vehicle can be designed separately. In this respect, the shock wave acts as a barrier in order to prevent spillage of higher-pressure airflow from the lower side of the vehicle to the upper side, resulting in a high-pressure differential and enhanced lift.

One of the key issues concerning hypersonic configurations is the leading edge of the vehicle. For flight at hypersonic speeds, the vehicle leading edges must be sufficiently blunt in order to reduce the heat transfer rate to acceptable levels, and possibly to allow for internal heat conduction. The use of blunt-nosed shapes 
tends to alleviate the aerodynamic heating problem since the heat flux for blunt bodies scales inversely with the square root of the nose radius at the stagnation point. Therefore, designing a hypersonic vehicle leading edge involves a tradeoff between making the leading edge sharp enough to obtain acceptable aerodynamic and propulsion efficiency and blunt enough to reduce the aerodynamic heating in the stagnation region.

Power law shaped leading edges $\left(y \propto x^{n}, 0<n<1\right)$ have been considered as especially promising bluntness for hypersonic configurations in order to provide the leading edge heating requirements. This concept is based on the work of Mason and $\mathrm{Lee}^{2}$, who have pointed out that, for certain exponents, power law shapes exhibit both blunt (infinite body slope at the nose) and sharp (zero radius of curvature at the nose) characteristics. Their analysis describes the details of the geometry and aerodynamics of low-drag axisymmetric bodies by using Newtonian theory. However, one of the important aspects of the problem, stagnation point heat transfer, was not considered.

The sensitivity of the pressure gradient and the stagnation point heating to shape variations of such leading edges has been investigated by Santos and Lewis ${ }^{3}$ for the idealized situation of two-dimensional rarefied hypersonic flow at zero angle of incidence. Through the use of Direct Simulation Monte Carlo (DSMC) method, they showed that the pressure gradient on the power law shapes in a rarefied environment is in agreement with that obtained by Mason and $\mathrm{Lee}^{2}$ by employing Newtonian analysis, i.e., $d C_{p} / d s \rightarrow-\infty$ as $x \rightarrow 0$. Santos and Lewis ${ }^{3}$ also found that the stagnation point heating behavior for power law leading edges with finite radius of curvature $(n=1 / 2)$ followed that predicted for classical blunt body, i.e., the heating rate on blunt bodies is inversely proportional to the square root of nose radius at the stagnation point. For those power law leading edges with zero radii of curvature $(n>1 / 2)$, the stagnation point heating is not a function of the curvature radius in the vicinity of the leading edges, but agrees with the classical blunt body behavior predicted by the continuum flow far from the stagnation point.

The knowledge of the flowfield structure and the aerodynamic surface quantities at zero angle of attack is not sufficient to predict with certainty the flow characteristics over these shapes with incidence. In this context, Santos and Lewis ${ }^{4}$ have investigated the essential characteristics of the angle of attack effect on both pressure gradient and stagnation point heating for power law bodies defined by exponents of $1 / 2,2 / 3$ and $3 / 4$, and positive angle of attack with 5,8 and 12 deg of incidence. It was found that, even though at incidence, the pressure gradient $d C_{p} / d s \rightarrow-\infty$ as $x \rightarrow 0$ along the both windward and leeward sides of the power law leading edges for the $n=3 / 4$ case. Thus, the leading edge with $n=3 / 4$ produces a flowfield that does not exhibit classical blunt body behavior, and can be considered as if it were sharp for the calculation of pressure distribution. It was also found that the heat flux to power law shaped leading edges followed that for classical blunt body far from the nose of the leading edges in that it is inversely proportional to the square root of the curvature radius. This dependence with the radius of curvature was more significant in the leeward side than that in the windward side with increasing the angle of attack, and it disappeared in the vicinity of the stagnation point.

Finally, O'Brien and Lewis ${ }^{5}$ have investigated power law shaped leading edges for possible use on hypersonic waverider vehicles. A numerical analysis was performed considering a two-dimensional inviscid flow. Results were compared to a corresponding circular cylinder to determine which geometry would be better suited as a blunting profile. Their analysis showed that circular cylinders provide smaller shock wave standoff distance and drag for equivalent stagnation point heating under the range of conditions investigated.

In an effort to obtain further insight into the nature of the flowfield structure of power law leading edges under hypersonic transitional flow conditions, a parametric study is performed on these shapes with a great deal of emphasis placed on the compressibility effects. In this scenario, the primary goal of this paper is to assess the sensitivity of the heat flux, total drag and shock wave standoff distance to variations in the freestream Mach number. The importance of this study is based on recent interest in hypersonic waveriders be considered for high-altitude/low-density applications $^{6-9}$.

For the transitional hypersonic flow, at high Mach number and high altitude, the flow departs from thermal equilibrium and the energy exchange into the various modes due to the vibrational excitation and relaxation becomes important. For the high altitude/high Knudsen number of interest, the flowfield is sufficient rarefied that continuum method is inappropriate. Alternatively, a Direct Simulation Monte Carlo (DSMC) method is used in the current study to calculate the rarefied hypersonic two-dimensional flow on the leading edge shapes.

\section{Leading Edge Geometry Definition}

In dimensional form, the body power law shapes are given by the following expression,

$$
y=a x^{n}
$$

where $n$ is the power law exponent and $a$ is the power law constant which is a function of $n$.

The power-law shapes are modeled by assuming a sharp leading edge of half angle $\theta$ with a circular cylinder of radius $R$ inscribed tangent to this wedge. The power law shapes, inscribed between the wedge and 
the cylinder, are also tangent to both shapes at the same common point where they have the same slope angle. The circular cylinder diameter provides a reference for the amount of blunting desired on the leading edges. It was assumed a leading edge half angle of 10 $\mathrm{deg}$, a circular cylinder diameter of $10^{-2} \mathrm{~m}$ and power law exponents of $1 / 2,0.6,2 / 3,0.7,3 / 4$ and 0.8 . Figure 1 shows schematically this construction.

From geometric considerations, the power law constant $a$ is obtained by matching slope on the wedge, circular cylinder and power law body at the tangency point. The common body height $H$ at the tangency point is equal to $2 R \cos \theta$, and the body length $L$ from the nose to the tangency point in the axis of symmetry is given by $n H / 2 \tan \theta$. Since the wake region behind the power law bodies is not of interest in this investigation, it was assumed that the power law bodies are infinitely long but only the length $L$ is considered.

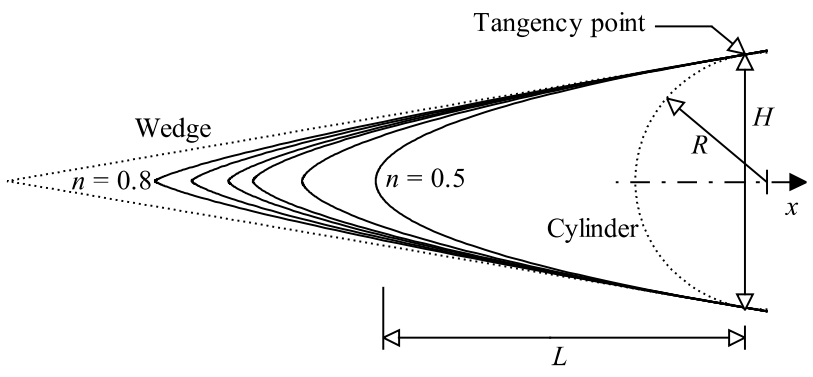

Fig. 1 Drawing illustrating the leading edge geometries.

\section{Computational Method and Procedure}

It is well known that neither the continuum flow equations nor the collisionless flow equations are valid to predict leading edge aerothermodynamics characteristics throughout the transitional flow regime. At this time it appears that the Direct Simulation Monte Carlo (DSMC) method ${ }^{10}$ is the most accurate and credible procedure for computing leading edge flowfield and surface effects in the transitional flow regime.

The DSMC method simulates real gas flows with various physical processes by means of a huge number of modeling particles, each of which is a typical representative of great number of real gas molecules. The DSMC method models the flow as being a collection of discrete particles, each one with a position, velocity and internal energy. The state of particles is stored and modified with time as the particles move, collide, and undergo boundary interactions in simulated physical space.

The molecular collisions are modeled by using the variable hard sphere (VHS) molecular model ${ }^{11}$. This model employs the simple hard sphere angular scattering law so that all directions are equally possible for post-collision velocity in the center-of-mass frame of reference. Nevertheless, the collision cross section depends on the relative speed of colliding molecules.
The energy exchange between kinetic and internal modes is controlled by the Borgnakke-Larsen statistical model $^{12}$. The essential feature of this model is that a fraction of the collisions is treated as completely inelastic, and the remainder of the molecular collisions is regarded as elastic. Simulations are performed using a non-reacting gas model consisting of two chemical species, $\mathrm{N}_{2}$ and $\mathrm{O}_{2}$. Energy exchanges between the translational and internal modes (rotation and vibration) are considered. The probability of an inelastic collision determines the rate at which energy is transferred between the translational and internal modes after an inelastic collision. For a given collision, the probabilities are designated by the inverse of the relaxation numbers, which correspond to the number of collisions necessary, on average, for a molecule to relax. For this study, the relaxation numbers of 5 and 50 were used for the rotation and vibration, respectively. The effective number of degrees of freedom in the partially excited vibrational states is calculated from the harmonic oscillator theory.

The flowfield is divided into a number of regions, which are subdivided into computational cells. The cell provides a convenient reference for the sampling of the macroscopic gas properties. The dimensions of the cells must be such that the change in flow properties across each cell is small. The linear dimensions of the cells should be small in comparison with the scale length of the macroscopic flow gradients in the streamwise directions, which means that the cell dimensions should be of the order of the local mean free path or even smaller ${ }^{10}$. The cells are further subdivided into 4 subcells, 2 subcells/cell in each direction. The collision partners are selected from the same subcell for the establishment of the collision rate. As a result, the flow resolution is much higher than the cell resolution. Time is advanced in discrete steps such that each step is small in comparison with the mean collision time ${ }^{10}$.

The computational domain used for the calculation is made large enough so that body disturbances do not reach the upstream and side boundaries, where freestream conditions are specified. A schematic view of the computational domain is depicted in Fig. 2. Side I is defined by the body surface. Diffuse reflection with complete thermal accommodation is the condition applied to this side. In this model, the reflection of the impinging molecules is not related to the preimpingement state of the molecules. The outgoing velocity of the molecules is randomly assigned according to a half-range Maxwellian distribution determined by the wall temperature. Advantage of the flow symmetry is taken into account, and molecular simulation is applied to one-half of a full configuration. Thus, side II is a plane of symmetry. In such a boundary, all flow gradients normal to the plane are zero. At the molecular level, this plane is equivalent to a specular reflecting boundary. Side III is the freestream 


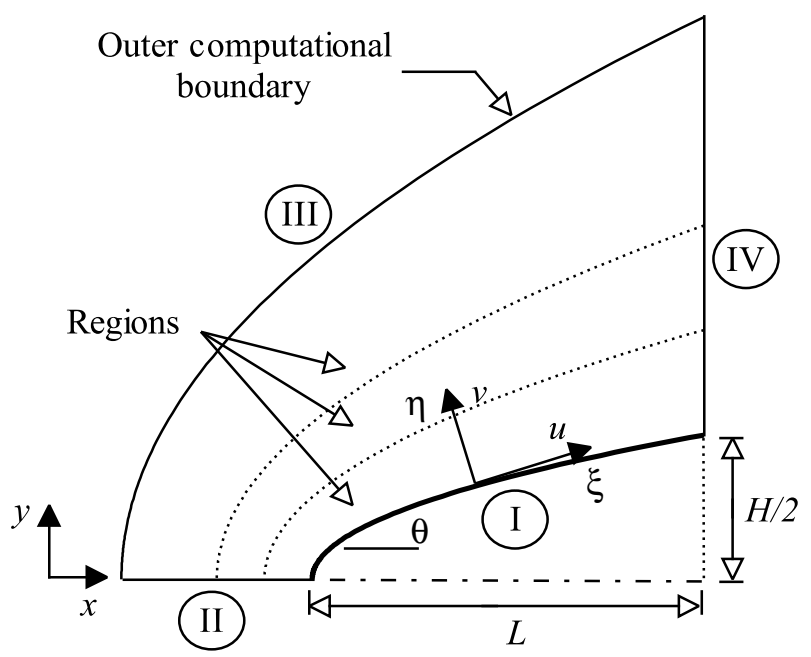

Fig. 2 Schematic view of the computational domain.

side through which simulated molecules enter and exit. Finally, the flow at the downstream outflow boundary, side IV, is predominantly supersonic and vacuum condition is specified ${ }^{10}$. At this boundary, simulated molecules can only exit.

Numerical accuracy in DSMC method depends on the grid resolution chosen as well as the number of particles per computational cell. Both effects were investigated to determine the number of cells and the number of particles required to achieve grid independence solutions for the thermal nonequilibrium flow that arises near the nose of the leading edges. A discussion of both effects on the aerodynamic surface quantities is described in the Appendix.

The conditions used for the numerical simulation of flow past the leading edges are those given by Santos ${ }^{13}$ and summarized in Table 1.

The freestream velocity $V_{\infty}$ assumed to be constant at $1.49,2.37$ and $3.56 \mathrm{~km} / \mathrm{s}$, corresponds to freestream Mach number $M_{\infty}$ of 5,8 and 12 , respectively. The temperature $T_{w}$ on the leading edge surface is maintained constant at $880 \mathrm{~K}$ for all cases considered. The overall Knudsen number is defined as the ratio of the molecular mean free path in the freestream gas to a characteristic dimension of the flowfield. In the present study, the characteristic dimension was defined as being the diameter of the reference circular cylinder. Therefore, the freestream Knudsen number $K n_{\infty}$ corresponds to 0.0903. The freestream Reynolds number by unit meter $R e_{\infty}$ covers the range from 8960 to 21420 , based on conditions in the undisturbed stream.

\section{Computational Results and Discussion}

Attention is now focused on the calculations of the heat transfer coefficient, total drag coefficient and the shock wave standoff distance obtained from the DSMC results. The purpose of this section is to discuss and to compare differences in the profiles of these properties
Table 1 Freestream and flow conditions

\begin{tabular}{lll}
\hline \hline Parameter & Value & Unit \\
\hline Altitude & 70 & $\mathrm{~km}$ \\
Temperature $\left(T_{\infty}\right)$ & 220.0 & $\mathrm{~K}$ \\
Pressure $\left(p_{\infty}\right)$ & 5.582 & $\mathrm{~N} / \mathrm{m}^{2}$ \\
Density $\left(\rho_{\infty}\right)$ & $8.753 \times 10^{-5}$ & $\mathrm{~kg} / \mathrm{m}^{3}$ \\
Viscosity $\left(\mu_{\infty}\right)$ & $1.455 \times 10^{-5}$ & $\mathrm{Ns} / \mathrm{m}^{2}$ \\
Number density $\left(n_{\infty}\right)$ & $1.8209 \times 10^{-21}$ & $\mathrm{~m}^{-3}$ \\
Mean free path $\left(\lambda_{\infty}\right)$ & $9.03 \times 10^{-4}$ & $\mathrm{~m}$ \\
Molecular weight & 28.96 & $\mathrm{~kg} / \mathrm{kg} . \mathrm{mol}$ \\
Molecular mass $O_{2}$ & $5.312 \times 10^{-26}$ & $\mathrm{~kg}$ \\
Molecular mass $N_{2}$ & $4.650 \times 10^{-26}$ & $\mathrm{~kg}$ \\
Molecular diameter $O_{2}$ & $4.01 \times 10^{-10}$ & $\mathrm{~m}$ \\
Molecular diameter $N_{2}$ & $4.11 \times 10^{-10}$ & $\mathrm{~m}$ \\
Mole fraction $O_{2}$ & 0.237 & \\
Mole fraction $N_{2}$ & 0.763 & \\
Viscosity index $O_{2}$ & 0.77 & \\
Viscosity index $N_{2}$ & 0.74 & \\
\hline \hline
\end{tabular}

due to variations on the freestream Mach number and on the leading edge shapes.

\section{Heat Transfer Coefficient}

The heat transfer coefficient $C_{h}$ is defined as follows,

$$
C_{h}=\frac{q_{w}}{\rho_{\infty} V_{\infty}^{3} / 2}
$$

where the heat flux $q_{w}$ to the body surface is calculated by the net energy flux of the molecules impinging on the surface. A flux is regarded as positive if it is directed toward the body surface. The net heat flux $q_{w}$ is related to the sum of the translational, rotational and vibrational energies of both incident and reflected molecules as defined by,

$$
\begin{array}{r}
q_{w}=q_{i}+q_{r}=\sum_{j=1}^{N}\left[\frac{1}{2} m_{j} c \prime_{j}^{2}+e_{R j}+e_{V j}\right]_{i}+ \\
\sum_{j=1}^{N}\left[\frac{1}{2} m_{j} c \prime_{j}^{2}+e_{R j}+e_{V j}\right]_{r}
\end{array}
$$

where $N$ is the number of molecules colliding with the surface by unit time and unit area, $m$ is the mass of the molecules, $c$ is the thermal velocity of the molecules, $e_{R}$ and $e_{V}$ stand for the rotational and vibrational energies, respectively. Subscripts $i$ and $r$ refer to incident and reflect molecules.

The compressibility effect on the heat transfer coefficient $C_{h}$ is illustated in Fig. 3 as a function of the dimensionless distance $S$ along the surface measured from the stagnation point. $S$ is the arc length $s$ normalized by the freestream mean free path $\lambda_{\infty}$.

According to the plots in Fig. 3, it is seen that the heat transfer coefficient changes not only with 
the power law exponents but also with the freestream Mach number. As the freestream Mach number increases from 5 to 12, the kinetic energy of the freestream molecules increases. As a result, the heat flux to the body surface increases. This behavior is better visualized in Eq.(3). The incident component of the thermal velocity of the molecules is a function of the freestream Mach number. However, the reflected component of the thermal velocity is not a function of the freestream Mach number. Due to the diffuse reflection model, the reflected component of the velocity is obtained from a Maxwellian distribution that only takes into account for the temperature of the body surface, which has the same value for the freestream Mach number range investigated. It also should be emphasized that the number of molecules colliding with the surface by unit time and unit area, which appears in Eq.(3), is the same for the incident and reflected components of the heat transfer coefficient. Nevertheless, it dramatically increases in the stagnation region of the leading edges with increasing the freestream Mach number (not shown) ${ }^{13}$. Referring to Figs. 3, it is also observed that the heat transfer coefficient is sensitive to the leading edge shapes. As would be expected, the blunter the leading edge is the lower the heat transfer coefficient to the body surface in the stagnation region. It is also apparent from the plots in Fig. 3 that there is a similarity among the heat transfer coefficient profiles. This is an indication that the heat transfer coefficient $C_{h}$ may shrink into one curve by employing appropriate functions. Nevertheless, no attempts have been done to find such functions.

\section{Total Drag Coefficient}

The drag on a surface in a gas flow results from the interchange of momentum between the surface and the molecules colliding with the surface. The total drag is obtained by the integration of the pressure $p_{w}$ and shear stress $\tau_{w}$ distributions along the body surface. In an effort to visualize the nature of the pressure and shear stress forces acting on the surface of the leading edges, both forces will be presented in this section.

The pressure $p_{w}$ on the body surface is calculated by the sum of the normal momentum fluxes of both incident and reflected molecules at each time step as follows,

$$
p_{w}=p_{i}+p_{r}=\sum_{j=1}^{N}\left\{\left[m_{j} c l_{\eta j}\right]_{i}+\left[m_{j} c l_{\eta j}\right]_{r}\right\}
$$

where $c^{\prime} \eta$ is the normal component of the thermal velocity of the molecules.

The sensitivity of the wall pressure, normalized by the freestream pressure $p_{\infty}$, to variations on the freestream Mach number is demonstrated in Fig. 4 for various for power law exponents. It is noted from the plots in Fig. 4 that the wall pressure presents the maximum value at the stagnation point and decreases

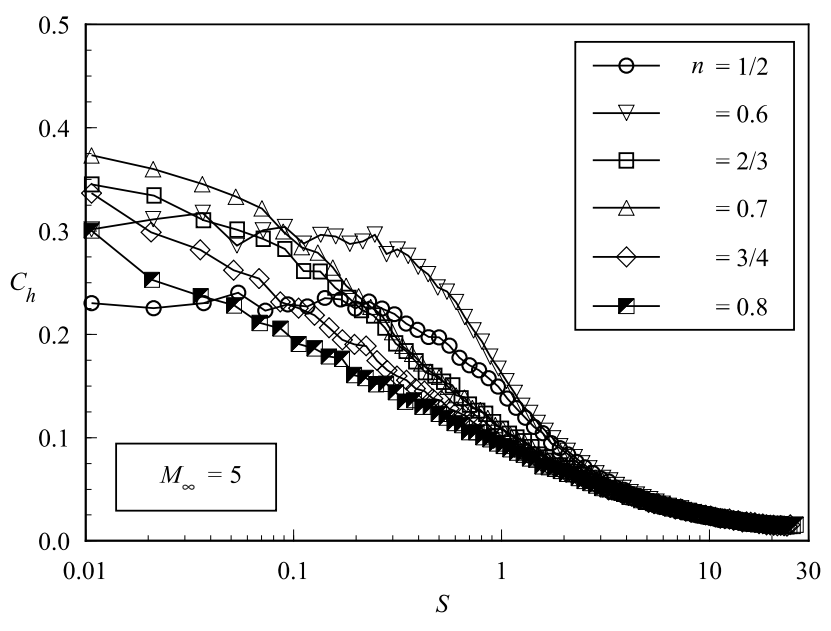

a) Freestream Mach Number of 5

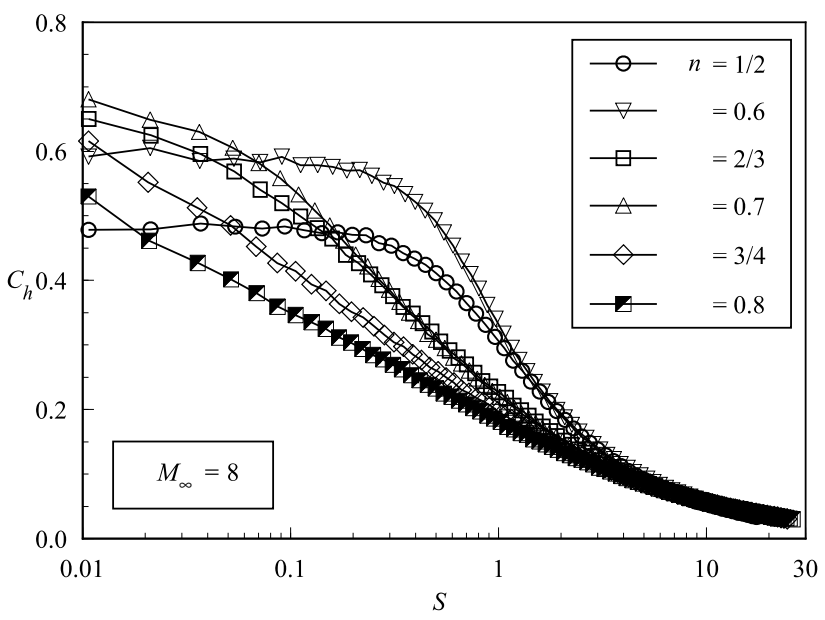

b) Freestream Mach Number of 8

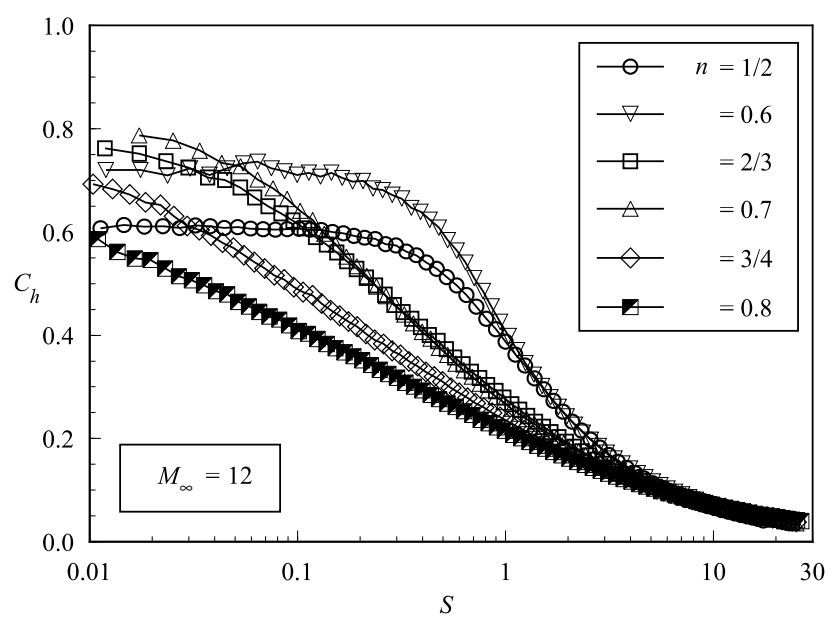

c) Freestream Mach Number of $\mathbf{1 2}$

Fig. 3 Distribution of heat transfer coefficient along the body surface for various power law bodies. 
fast along the body surface as the power law exponent increases. Also, the leading edges experience a remarkable pressure rise with increasing the freestream Mach number, as would be expected.

The shear stress $\tau_{w}$ on the body surface is calculated by averaging the tangential momentum transfer of the molecules impinging on the surface. For the diffuse reflection model imposed for the gas-surface interaction, reflected molecules have a tangential moment equal to zero, since the molecules essentially lose, on average, their tangential velocity component. In this fashion, the tangential momentum flux of the incident molecules is defined by the following expression,

$$
\tau_{w}=\sum_{j=1}^{N} m_{j} c^{\prime} \xi j
$$

where $c_{\xi}$ is the tangential component of the thermal velocity of the molecules.

Distributions of the shear stress, normalized by the freestream pressure $p_{\infty}$, along the body surface at different freestream Mach number are displayed in Fig. 5 with the power law exponent as a parameter.

Referring to Fig. 5, it is observed that the shear stress increases as the freestream Mach number increases. This trend is as expected since the thermal velocity of the molecules increases with increasing the freestream Mach number, as was pointed out earlier.

The total drag coefficient is defined as being,

$$
C_{d}=\frac{D}{\rho_{\infty} V_{\infty}^{2} H / 2}=\frac{D / p_{\infty}}{\gamma M_{\infty}^{2} H / 2}
$$

where $D$ is the resultant force acting on the body surface, $\gamma$ is the specific heat ratio and $H$ is the height at the matching point common to the leading edges (see Fig. 1).

The resultant force acting on the body surface was obtained by the integration of the pressure $p_{w}$ and shear stress $\tau_{w}$ distributions from the stagnation point of the leading edges to the station $L$ that corresponds to the tangential point common to all shapes. It is important to mention that the values for the total drag coefficient were obtained by assuming the shapes acting as leading edges. As a result, no base pressure effects were taken into account on the calculations. The DSMC results for total drag are presented as total drag coefficient $C_{d}$ and its components of pressure drag coefficient $C_{p d}$ and skin friction drag coefficient $C_{f d}$ on Tables 2, 3 and 4 for freestream Mach number of 5,8 and 12 , respectively.

According to this set of Tables, it is apparent that as $n \rightarrow 0.8$, the pressure drag contribution to the total drag coefficient decreases. In contrast, as $n \rightarrow 0.8$, the skin friction drag contribution increases. Also, it is seen that the pressure drag contribution diminishes and the skin friction drag contribution increases with increasing the freestream Mach number. As the

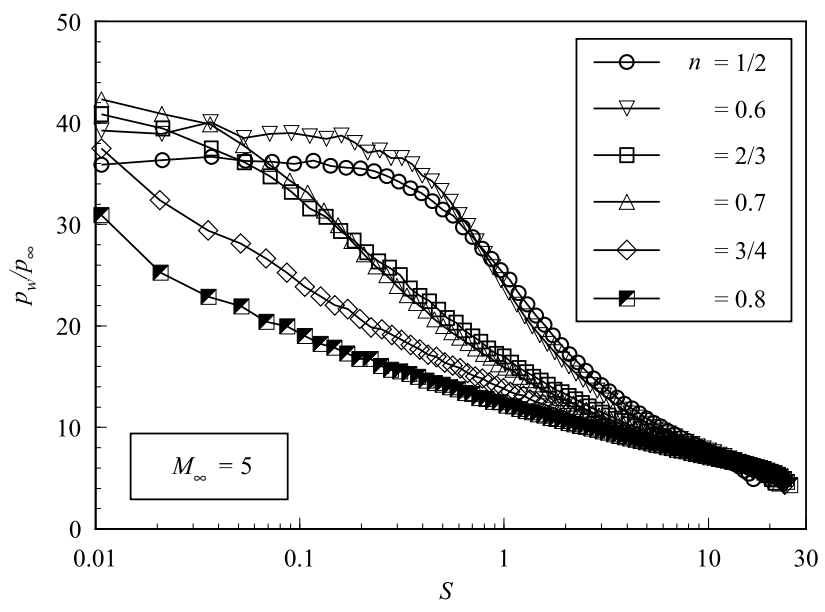

a) Freestream Mach Number of 5

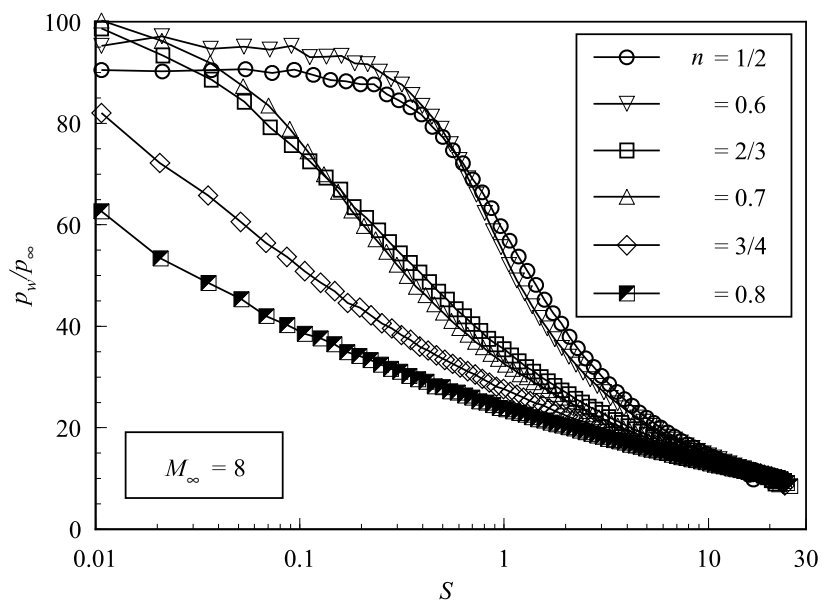

b) Freestream Mach Number of 8

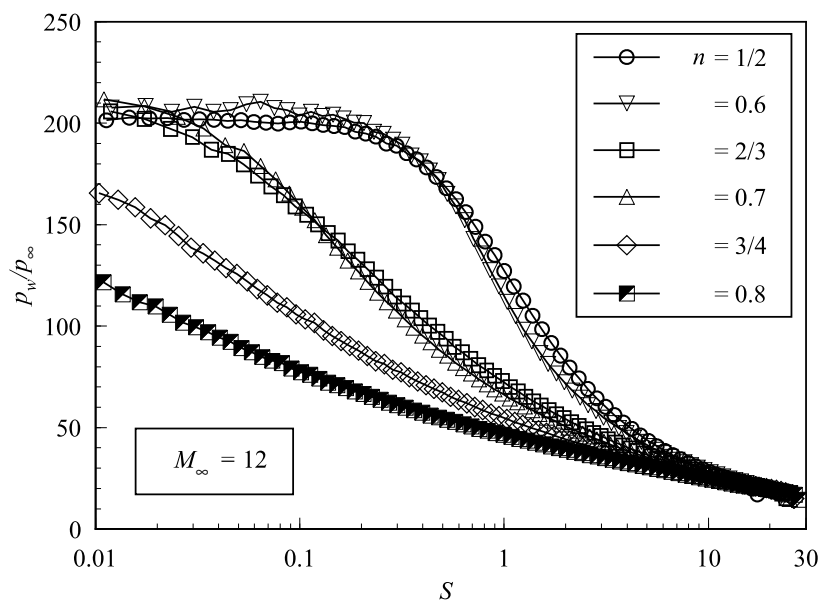

c) Freestream Mach Number of 12

Fig. 4 Distribution of dimensionless wall pressure along the body surface for various power law bodies. 


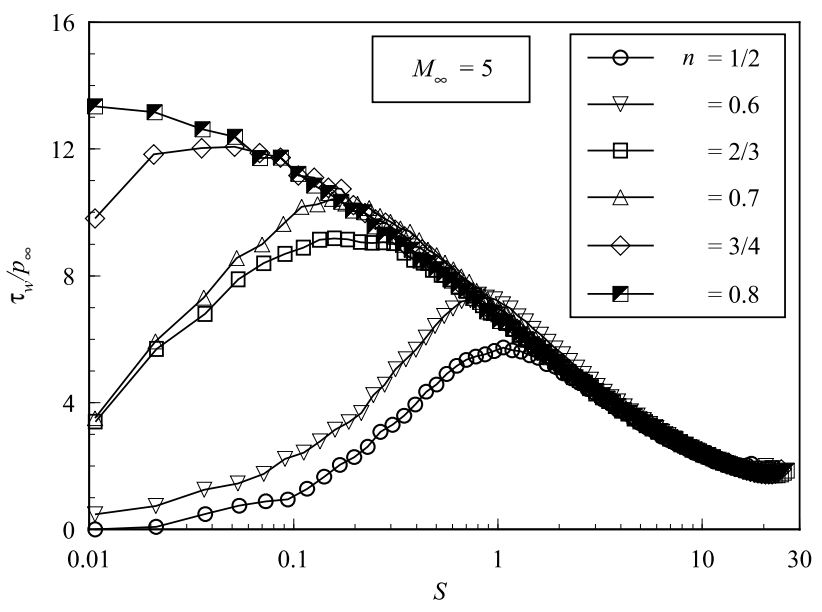

a) Freestream Mach Number of 5

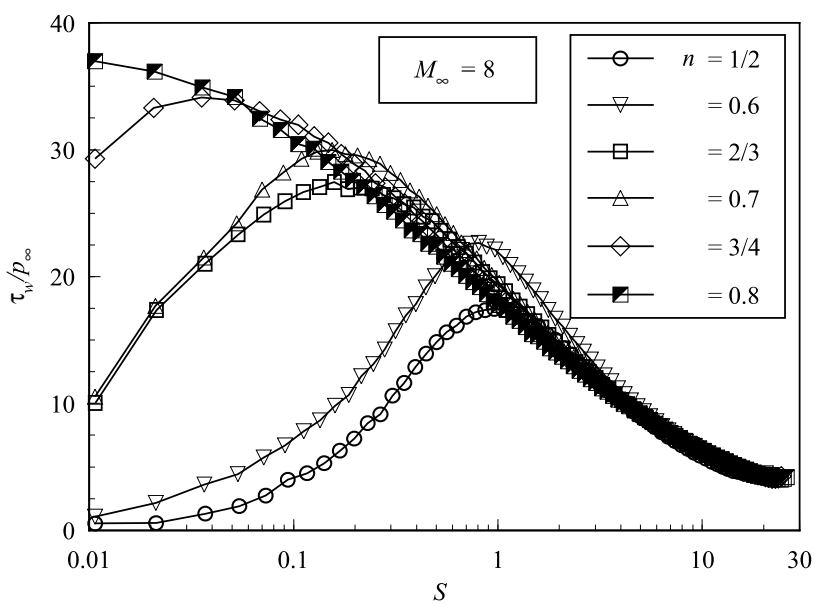

b) Freestream Mach Number of 8

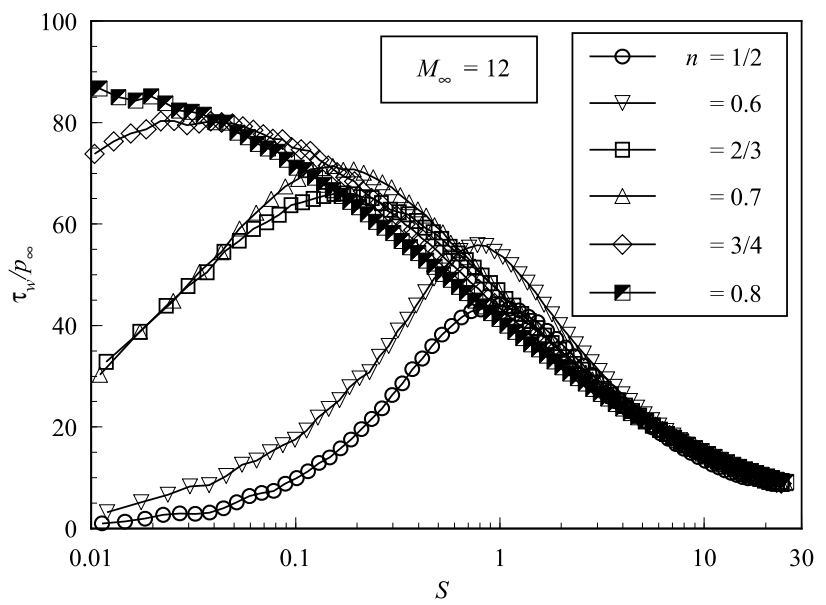

c) Freestream Mach Number of 12

Fig. 5 Distribution of dimensionless shear stress along the body surface for various power law bodies.
Table 2 Pressure drag, skin friction drag and total drag coefficient for $M_{\infty}=5$.

\begin{tabular}{cccccc}
\hline \hline$n$ & $C_{p d}$ & $C_{f d}$ & $C_{d}$ & $\% C_{p d} / C_{d}$ & $\% C_{f d} / C_{d}$ \\
\hline $1 / 2$ & 0.829 & 0.465 & 1.295 & 64.0 & 36.0 \\
0.6 & 0.816 & 0.567 & 1.383 & 59.0 & 41.0 \\
$2 / 3$ & 0.587 & 0.618 & 1.205 & 55.9 & 44.1 \\
0.7 & 0.568 & 0.650 & 1.218 & 46.6 & 53.4 \\
$3 / 4$ & 0.491 & 0.683 & 1.175 & 41.8 & 58.2 \\
0.8 & 0.446 & 0.715 & 1.161 & 38.4 & 61.6 \\
\hline \hline
\end{tabular}

Table 3 Pressure drag, skin friction drag and total drag coefficient for $M_{\infty}=8$.

\begin{tabular}{cccccc}
\hline \hline$n$ & $C_{p d}$ & $C_{f d}$ & $C_{d}$ & $\% C_{p d} / C_{d}$ & $\% C_{f d} / C_{d}$ \\
\hline $1 / 2$ & 0.714 & 0.478 & 1.196 & 59.7 & 40.3 \\
0.6 & 0.671 & 0.592 & 1.275 & 52.6 & 47.4 \\
$2 / 3$ & 0.444 & 0.637 & 1.099 & 40.4 & 59.6 \\
0.7 & 0.423 & 0.669 & 1.110 & 38.1 & 61.9 \\
$3 / 4$ & 0.353 & 0.697 & 1.066 & 33.1 & 66.9 \\
0.8 & 0.313 & 0.723 & 1.050 & 29.8 & 70.2 \\
\hline \hline
\end{tabular}

Table 4 Pressure drag, skin friction drag and total drag coefficient for $M_{\infty}=12$.

\begin{tabular}{cccccc}
\hline \hline$n$ & $C_{p d}$ & $C_{f d}$ & $C_{d}$ & $\% C_{p d} / C_{d}$ & $\% C_{f d} / C_{d}$ \\
\hline $1 / 2$ & 0.672 & 0.505 & 1.176 & 57.1 & 42.9 \\
0.6 & 0.628 & 0.626 & 1.254 & 50.0 & 60.0 \\
$2 / 3$ & 0.410 & 0.668 & 1.078 & 38.0 & 62.0 \\
0.7 & 0.389 & 0.699 & 1.088 & 35.8 & 64.2 \\
$3 / 4$ & 0.320 & 0.724 & 1.044 & 30.6 & 69.4 \\
0.8 & 0.280 & 0.747 & 1.027 & 27.3 & 72.7 \\
\hline \hline
\end{tabular}

net effect on total drag depends on opposite behavior between pressure drag and skin friction drag, hence appreciable changes are observed in the total drag coefficient for the power law exponent and for the freestream Mach number range investigated.

The extent of the changes in the total drag coefficient $C_{d}$ with increasing the freestream Mach number is demonstrated in Fig. 6. Significant differences in total drag coefficient on the leading edge shapes are observed for the freestream Mach number range investigated. Referring to Fig. 6, the total drag coefficient is reduced as the freestream Mach number increases, despite of the fact that the wall pressure and shear stress increase significantly with increasing freestream Mach number, as shown in Figs. 4 and 5. Equation (6) provides the necessary assistance in order to understand this behavior. The numerator of Eq.(6) grows with wall pressure and shear stress, while the denom- 


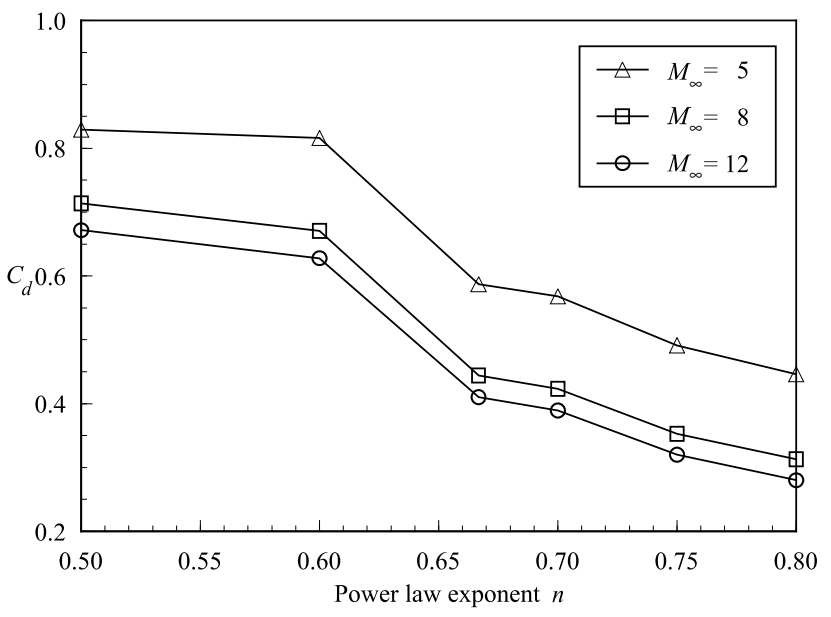

Fig. 6 Drag coefficient as a function of the body power law exponent $n$.

inator $\left(\propto M_{\infty}^{2}\right)$ increases faster than the numerator and results in a total drag coefficient decrease.

\section{Shock Wave Standoff Distance}

The problem of predicting the shape and location of detached shock waves has been stimulated by the necessity for blunt noses and leading edges configurations designed for hypersonic flight in order to cope with the aerodynamic heating. Also, the ability to predict the shape and location of shock waves is of primary importance in analysis of aerodynamic interference. In addition, displacement of the shock wave is especially undesirable in a waverider geometry, because these hypersonic configurations usually depend on shock wave attachment at the leading edge to achieve their high lift-to-drag ratio at high-lift coefficient. In this context, the intent in this section is to present the shock wave standoff distance obtained from the numerical simulations.

In a rarefied flow, the shock wave has a finite region that depends on the transport properties of the gas, and can no longer be considered as a discontinuity obeying the classical Rankine-Hugoniot relations. In this scenario, the shock standoff distance is defined as being the distance between the shock wave center and the nose of the leading edge along the stagnation streamline.

In order to quantify the shock standoff distance, the shock wave center location is determined by employing the following procedure: the flow is assumed to consist of three distinct classes of molecules; those molecules from the freestream that have not been affected by the presence of the leading edge are denoted as class I molecules; those molecules that, at some time in their past history, have struck and been reflected from the body surface are denoted as class II molecules; and those molecules that have been indirectly affected by the presence of the body are defined as class III molecules. Figure 7 illustrates the classification of the molecules.

It is assumed that the class I molecule changes to class III molecule when it collides with class II or class III molecule. Class I or class III molecule is progressively transformed into class II molecule when it interacts with the body surface. Also, a class II molecule remains class II regardless of subsequent collisions and interactions. Hence, the transition from class I molecules to class III molecules may represent the shock wave, and the transition from class III to class II may define the boundary layer.

The molecule classification that has been adopted here was presented by Lubonski ${ }^{14}$ in order to study the hypervelocity Couette flow near the free molecule regime. He divided the gas into three classes of molecules: "freestream", "reflected from the boundary" and "scattered". For the purpose of flow visualization, Bird ${ }^{15}$ applied the same scheme of classification by identifying the classes by colors: blue for class I, red for class II and yellow for class III molecules.

For illustration purpose, the distribution of molecules for each class along the stagnation streamline is demonstrated in Fig. 8 for cases $n=1 / 2$ and 0.8 and freestream Mach number of $12 . \eta / \lambda_{\infty}$ is the dimensionless distance away from the body (see Fig. 2), and $f$ is the ratio of the number of molecules for each one of the classes to the total amount of molecules inside each cell. Hence, the shock wave center location is obtained by calculating the position that corresponds to the maximum value $f$ for class III molecules.

The shock wave standoff distance $\Delta$, defined as being the distance from the shock wave center to the nose of the leading edge along the stagnation streamline, can be observed in Fig. 8. Table 5 displays the shock wave standoff distance, normalized by $\lambda_{\infty}$, for all cases investigated, and Fig. 9 depicts the dimen-

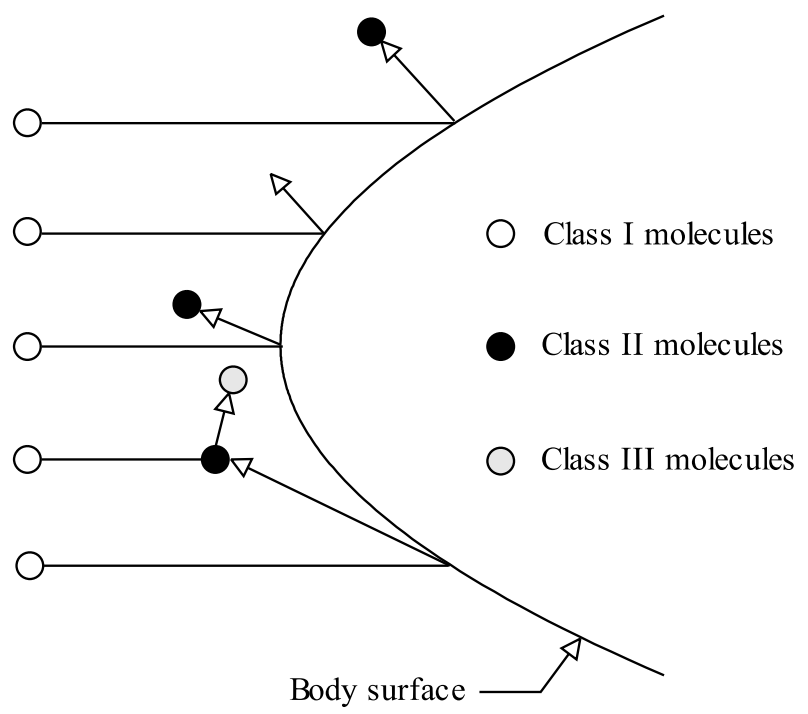

Fig. 7 Drawing illustrating the classification of molecules. 


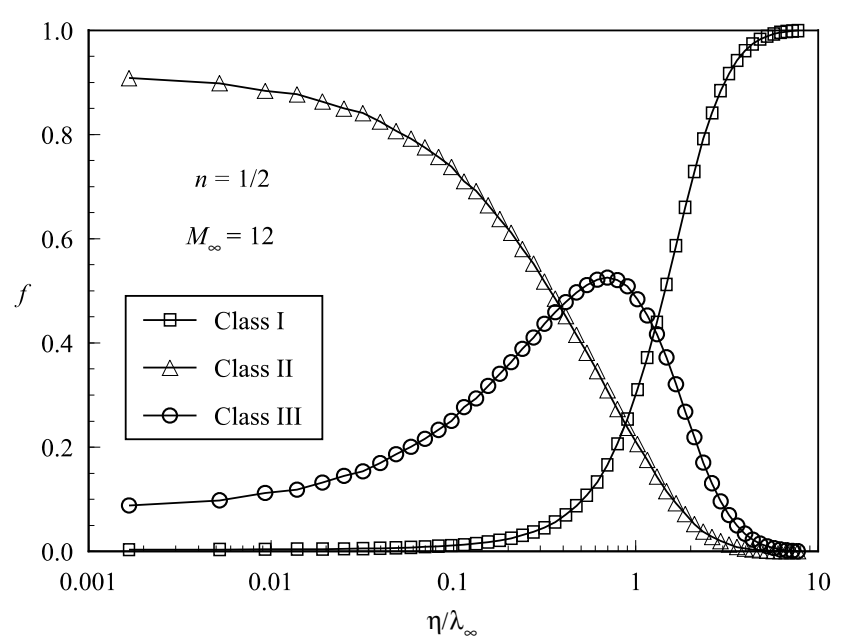

a) Power law exponent of $1 / 2$

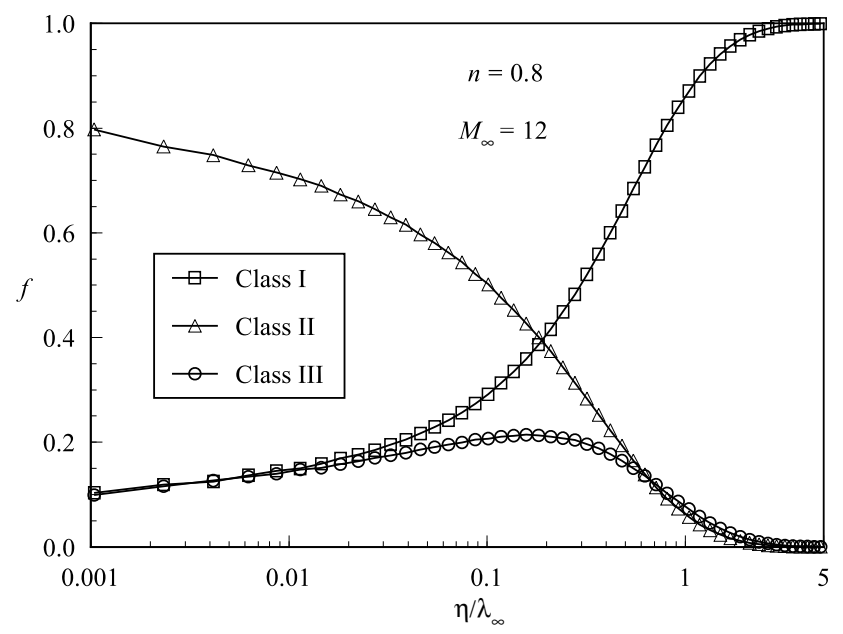

b) Power law exponent of 0.8

Fig. 8 Distributions of molecules for classes I, II and III along the stagnation streamline.

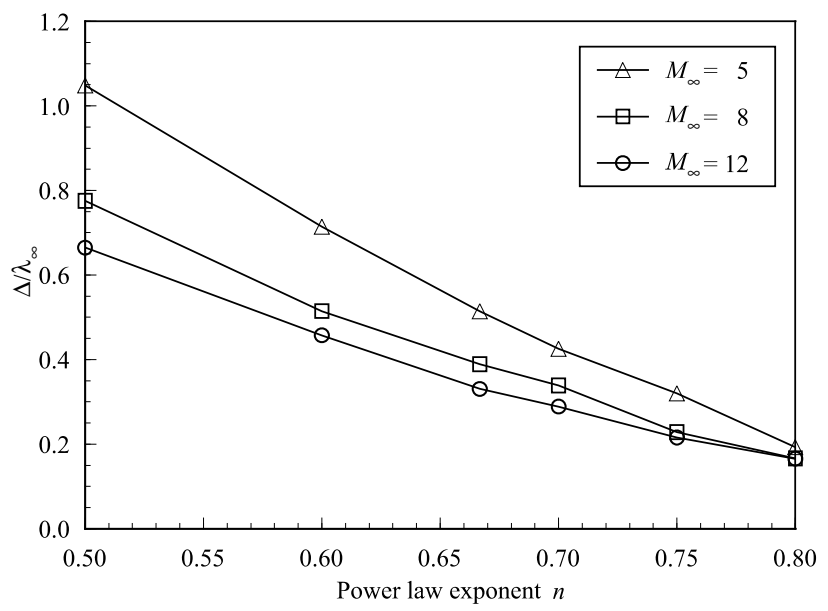

Fig. 9 Dimensionless shock standoff distance as function of the freestream Mach number for various power law shapes.
Table 5 Dimensionless shock wave standoff distance $\Delta / \lambda_{\infty}$ for freestream Mach number of 5, 8 and 12 .

\begin{tabular}{cccc}
\hline \hline$n$ & $M_{\infty}=5$ & $M_{\infty}=8$ & $M_{\infty}=12$ \\
\hline $1 / 2$ & 1.048 & 0.775 & 0.665 \\
0.6 & 0.714 & 0.515 & 0.457 \\
$2 / 3$ & 0.514 & 0.389 & 0.330 \\
0.7 & 0.425 & 0.339 & 0.289 \\
$3 / 4$ & 0.320 & 0.228 & 0.216 \\
0.8 & 0.193 & 0.166 & 0.165 \\
\hline \hline
\end{tabular}

sionless standoff distance $\Delta / \lambda_{\infty}$ as a function of the freestream Mach number. As observed in Fig. 9, there is a discrete shock standoff distance for all cases investigated. The results tend to confirm the expectation that the shock standoff distance decreases not only with increasing the freestream Mach number but also with increasing the power law exponent $n$, i.e., as the leading edge becomes aerodynamically sharp.

\section{Concluding Remarks}

This study applies the Direct Simulation Monte Carlo method to investigate rarefied gas over power law shaped leading edges. Effects of air speed on the heat transfer coefficient, drag coefficient and shock wave standoff distance for a wide range of parameters are investigated. The freestream Mach number is varied from 5 to 12 , and the power law exponent ranges from $1 / 2$ to 0.8 . Cases considered in this study cover the hypersonic flow on the transitional regime.

Calculations showed that an increase in the freetream Mach number from 5 to 12 increased the heat transfer coefficient for the power law shapes investigated. Also, it was observed a significant decrease on the total drag coefficient and on the shock wave standoff distance with increasing the freestream Mach number.

\section{References}

1 Nonweiler, T. R. F.,"Aerodynamic Problems of Manned Space Vehicles," J. of the Royal Aeronautical Society, vol. 63, Sept, 1959, pp.521-528.

2 Mason, W. H. and Lee, J.,"Aerodynamically Blunt and Sharp Bodies," J. of Spacecraft and Rockets, vol. 31, No. 3, 1994, pp.378-382.

3 Santos, W. F. N., and Lewis, M. J., "Power Law Shaped Leading Edges in Rarefied Hypersonic Flow," J. of Spacecraft and Rockets, vol. 39, No. 6, 2002, pp.917-925.

4 Santos, W. F. N., and Lewis, M. J., "Angle of Attack Effect on Rarefied Hypersonic Flow over 
Power Law Shaped Leading Edges," in 23rd International Symposium on Rarefied Gas Dynamics, Whistler, BC, Canada, 20-25 July 2002.

5 O'Brien, T. F. and Lewis, M. J., "Power Law Shapes for Leading-Edge Blunting with Minimal Shock Standoff," J. of Spacecraft and Rockets, vol. 36, No. 5, 1999, pp.653-658.

${ }^{6}$ Anderson, J. L., "Tethered Aerothermodynamic Research for Hypersonic Waveriders," in Proceedings of the 1st International Hypersonic Waverider Symposium, Univ. of Maryland, College Park, MD, 1990.

7 Potter, J. L. and Rockaway, J. K., "Aerodynamic Optimization for Hypersonic Flight at Very High Altitudes," in Rarefied gas Dynamics: Space Science and Engineering, edited by B. D. Shizgal and D. P. Weaver, Vol. 160, Progress in Astronautics and Aeronautics, AIAA New York, 1994, pp.296307.

8 Rault, D. F. G., "Aerodynamic Characteristics of a Hypersonic Viscous Optimized Waverider at High Altitude," J. of Spacecraft and Rockets, vol. 31, No. 5, 1994, pp.719-727.

9 Graves, R. E. and Argrow, B. M.," Aerodynamic Performance of an Osculating-Cones Waverider at High Altitudes," in 35th AIAA Thermophysics Conference, Paper AIAA 2001-2960, Anaheim, CA, 2001.

10 Bird, G. A.,Molecular Gas Dynamics and the Direct Simulation of Gas Flows, Oxford University Press, Oxford, England, UK, 1994.

11 Bird, G. A., "Monte Carlo Simulation in an Engineering Context," in Progress in Astronautics and Aeronautics: Rarefied gas Dynamics, edited by Sam S. Fisher, Vol. 74, part I, AIAA New York, 1981, pp.239-255.

12 Borgnakke, C. and Larsen, P. S., "Statistical Collision Model for Monte Carlo Simulation of Polyatomic Gas Mixture," Journal of computational Physics, vol. 18, No. 4, 1975, pp.405-420.

13 Santos, W. F. N., "Direct Simulation Monte Carlo of Rarefied Hypersonic Flow on Power Law Shaped Leading Edges," Ph.D. Dissertation, Dept. of Aerospace Engineering, University of Maryland, College Park, MD, Dec., 2001.

${ }^{14}$ Lubonski, J., "Hypersonic Plane Couette Flow in Rarefied Gas," in Archiwum Mechaniki Stosowanej, vol. 14, No. 3/4, 1962, pp.553-560.

15 Bird, G. A., "The Structure of Rarefied Gas Flows Past Simple Aerodynamic Shapes," in J. of Fluid Mechanics, vol. 36, No. 3, 1969, pp.571-576.

\section{Appendix}

This section focuses on the analysis of the influence of the cell size and the number of particles per computational cell on the surface properties in order to achieve grid independence solutions. The analysis is limited to the case defined by $n=1 / 2$ and $M_{\infty}=12$.

\section{Effect of Grid Variation}

The effect of grid resolution on computed results is of particular interest for the present study since insufficient grid resolution can reduce significantly the accuracy of the predicted aerodynamic heating and forces acting on the body surface. Hence, heat transfer, pressure and skin friction coefficients are used as the representative parameters for the grid sensitivity study.

The effect of altering the size of the computational cells is investigated for a series of three simulations with grids of 35(coarse), 70(standard) and 105(fine) cells in the $\xi$-direction and 50 cells in the $\eta$-direction (see Fig. 2). Each grid was made up of nonuniform cell spacing in both directions. The effect of changing the number of cells in the $\xi$-direction is illustrated in Fig. 10 as it impacts the calculated heat transfer and skin friction coefficients, and in Fig. 11 as it impacts the pressure coefficient. The comparison shows that the calculated results are rather insensitive to the range of cell spacing considered.

In analogous fashion, an examination was made in the $\eta$-direction. The sensitivity of the calculated results to cell size variations in the $\eta$-direction is displayed in Fig. 12 for heat transfer and skin friction coefficients, and in Fig. 13 for pressure coefficient. In this set of Figs., a new series of three simulations with grid of 70 cells in the $\xi$-direction and 25 (coarse), 50 (standard) and 75 (fine) cells in the $\eta$-direction is compared. The cell spacing in both directions is again nonuniform. According to Figs. 12 and 13, the results

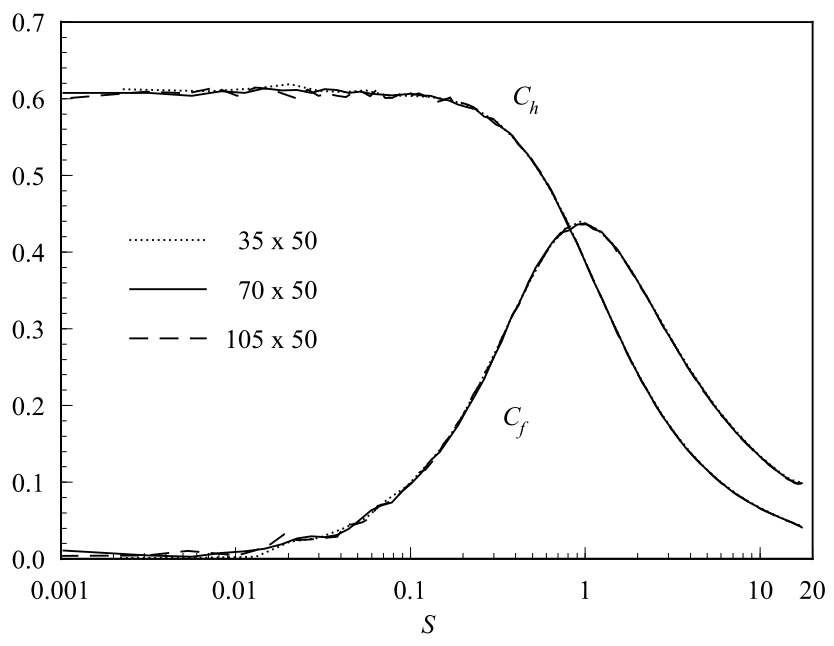

Fig. 10 Effect of altering the cell size in the $\xi$-direction on skin friction and heat transfer coefficients. 


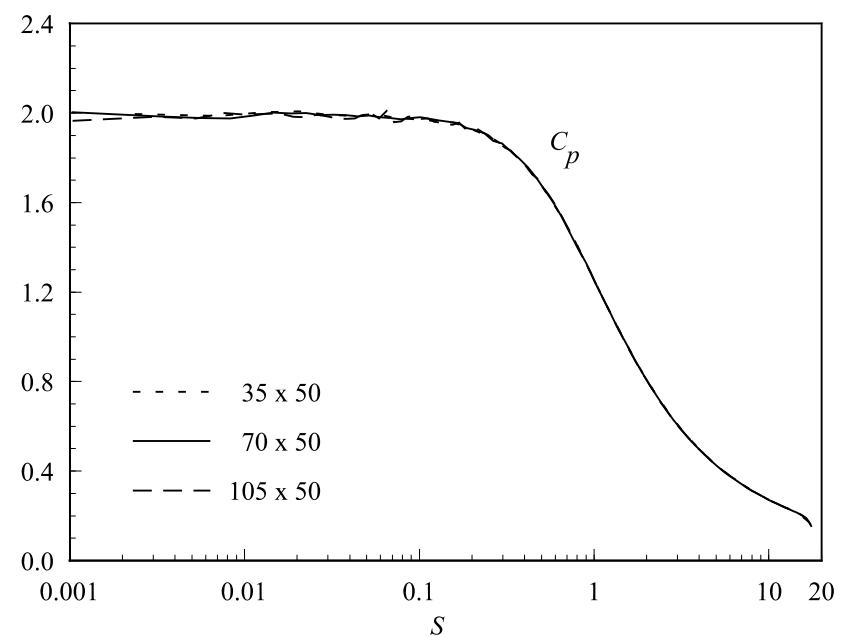

Fig. 11 Effect of altering the cell size in the $\xi$ direction on pressure coefficient.

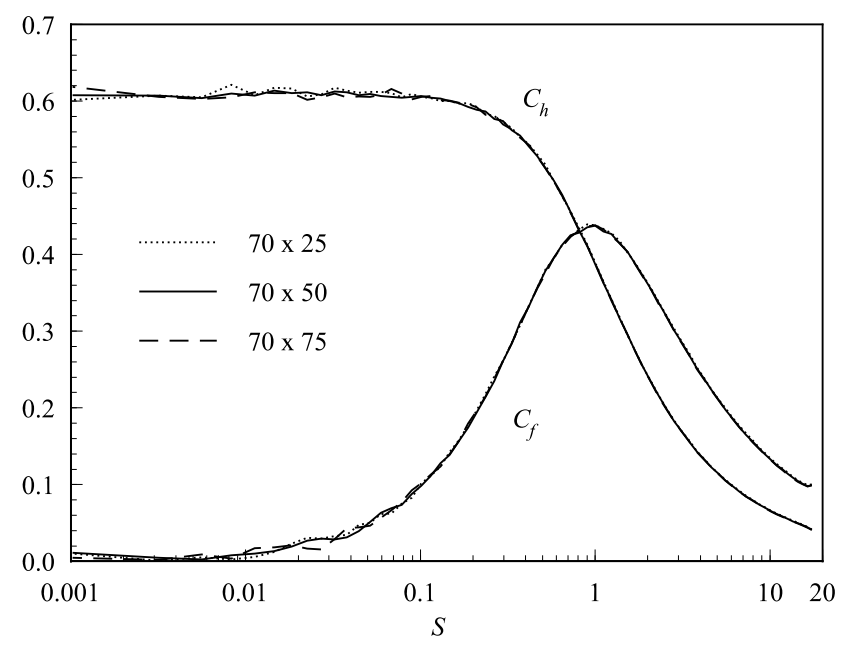

Fig. 12 Effect of altering the cell size in the $\eta$-direction on skin friction and heat transfer coefficients.

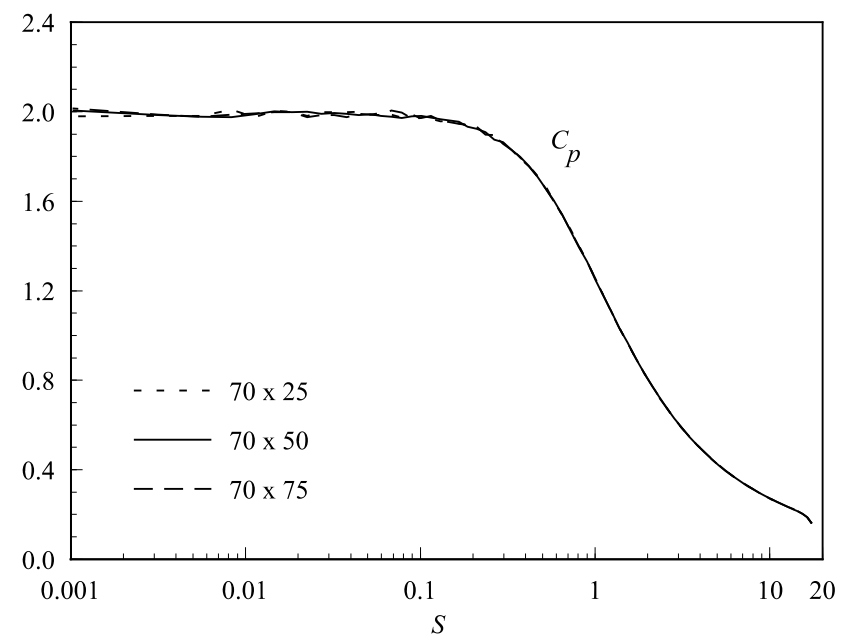

Fig. 13 Effect of altering the cell size in the $\eta$ direction on pressure coefficient.

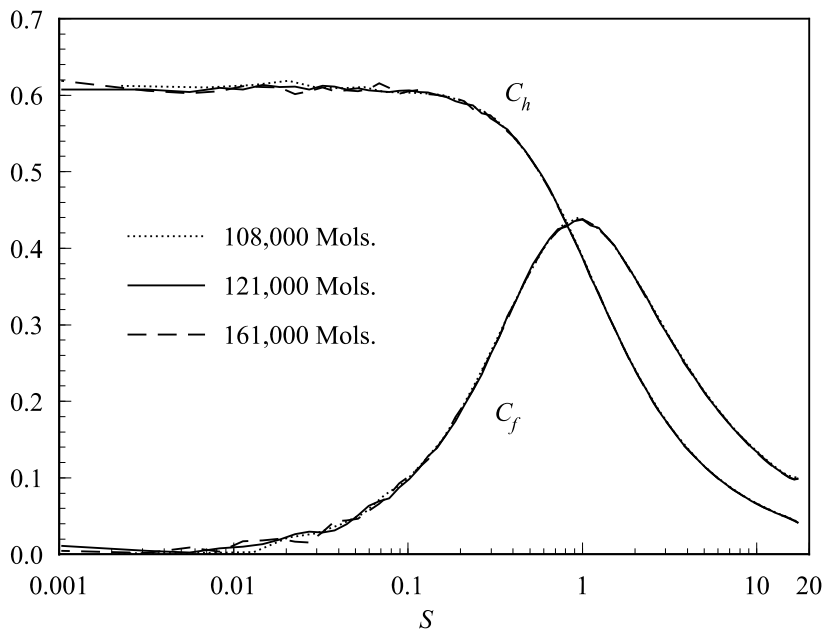

Fig. 14 Effect of altering the number of molecules on skin friction and heat transfer coefficients.

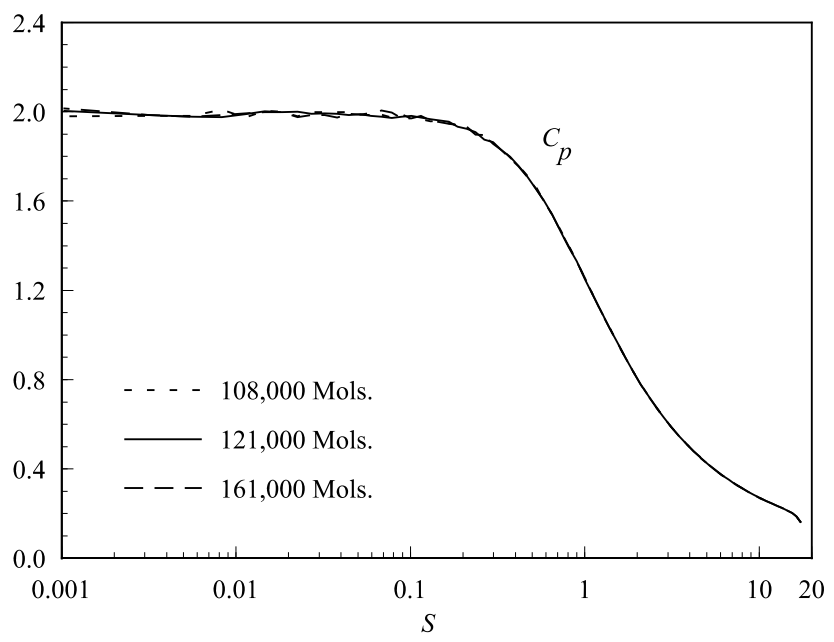

Fig. 15 Effect of altering the number of molecules on pressure coefficient.

for the three grids are approximately the same, indicating that the standard grid, 70x50 cells, is essentially grid independent. For the standard case, the cell size in the $\eta$-direction is always less than the local mean free path length in the vicinity of the surface.

\section{Effect of Number of Molecules}

A similar examination was made for the number of molecules. The sensitivity of the calculated results to number of molecule variations is demonstrated in Fig. 14 for heat transfer and skin friction coefficients, and in Fig. 15 for pressure coefficient. The standard grid, 70x50 cells, corresponds to a total of 121,000 molecules. Two new cases using the same grid were investigated. These new cases correspond to, on average, 108,000 and 161,000 molecules in the entire computational domain. It is seen that the standard grid with a total of 121,000 molecules is enough for the computation of the aerodynamic surface quantities. 